\title{
Overwhelming Asplenic Sepsis due to Babesiosis
}

\author{
Babesiyozis Illişkili Ağır Asplenik Sepsis
}

(1) Chakra P. Chaulagain

Department of Hematology-Oncology, Myeloma and Amyloidosis Program, Maroone Cancer Center, Cleveland Clinic Florida, Weston, FL, USA

\section{To the Editor,}

A 70-year-old female from southern Massachusetts, USA, was admitted to the intensive care unit with septic shock and acute respiratory distress syndrome (ARDS) after 3 days of acute febrile illness. She had undergone splenectomy at the age of 5 related to trauma from a traffic accident. Laboratory studies reveled pancytopenia, acute renal insufficiency, increased lactate dehydrogenase, depressed haptoglobin, and elevated liver enzymes with indirect hyperbilirubinemia. Prothrombin time and activated partial thromboplastin time were both elevated and fibrinogen level was low, consistent with disseminated intravascular coagulation (DIC). A direct anti-globulin test was negative. A thin blood smear with oil immersion showed intraerythrocytic polymorphic ring forms (Figure 1, arrows) morphologically consistent with Babesia species and the presence of Howell-Jolly bodies (Figure 1, arrowhead), confirming the history of splenectomy. Real-time DNA-PCR confirmed Babesia microti as the offending parasite. The patient was started on treatment for babesiosis with quinine, azithromycin, and atovaquone. She also received red cell exchange transfusion due to the high level of parasitemia (14\% of the erythrocytes) and completely recovered in the next few weeks.

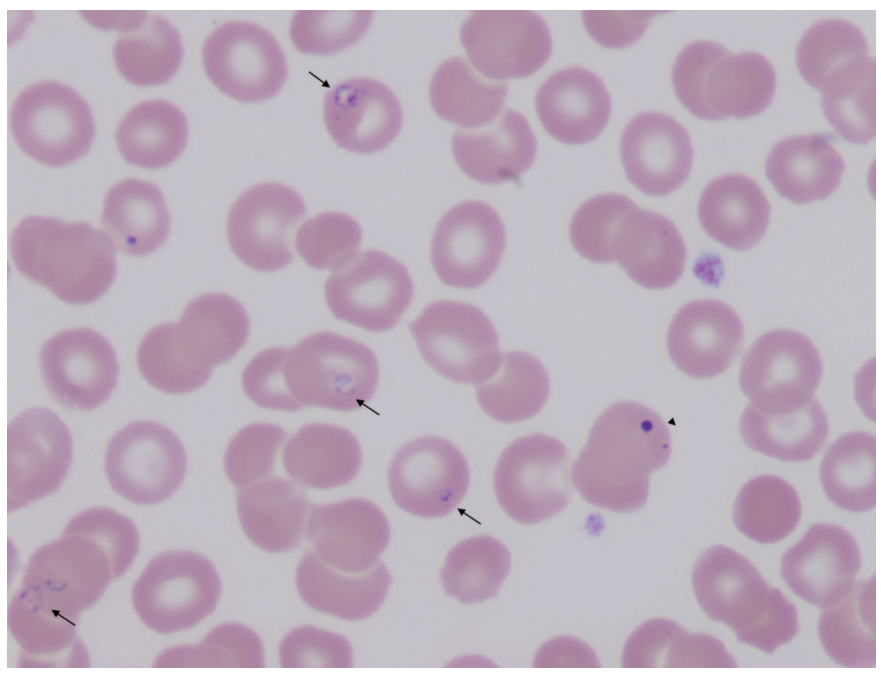

Figure 1. Thin blood smear with oil immersion showed intraerythrocytic polymorphic ring forms (arrows) morphologically consistent with Babesia species and the presence of Howell-Jolly bodies (arrowhead), confirming the history of splenectomy.
Human babesiosis is a malaria-like tick-borne illness caused by the protozoan parasite Babesia microti, endemic in the Midwest and Northeast USA; it has also been reported in parts of Europe, Asia, and Australia [1]. It has also been reported after transfusion of contaminated blood products [2]. Infection is usually mild to moderate in an immunocompetent host but a severe infection requiring hospitalization can occur in patients with a history of splenectomy or immunodeficiency such as cancer, human immunodeficiency virus infection, or hemoglobinopathy and in the elderly with co-morbidities and allogeneic hematopoietic stem cell transplant recipients $[1,3]$. Severe babesiosis with ARDS and DIC can occur in immunocompromised or asplenic individuals, which can be fatal $[4,5]$. Milder illness in immunocompetent hosts manifests with malaise, fever, headache, myalgia, and nausea. Laboratory findings typically show non-immune hemolytic anemia and thrombocytopenia, but immune hemolytic anemia has also been reported. A rapid diagnosis can be made by identification of Babesia organisms on thin blood smears under oil immersion. The diagnosis can be confirmed by using DNA-PCR to identify the DNA of the parasite. Serology is available, but it is difficult to distinguish current from recent or past infection in a patient coming from an endemic area. The most commonly used agents for treatment of severe babesiosis include azithromycin, atovaquone, quinine, and clindamycin. Patients with severe infection with highgrade parasitemia, severe hemolysis, or compromised organ functions (pulmonary, liver, or renal impairment) may benefit from red cell exchange transfusion.

This case confirms that a severe form of babesiosis can occur in patients who have undergone splenectomy. A high index of suspicion and a timely review of blood smears in asplenic patients presenting with febrile illness and hemolytic anemia from endemic areas can aid in rapid diagnosis and prompt treatment, which can be lifesaving. This case illustrates that an early diagnosis and aggressive treatment can be lifesaving even with a fulminant and severe infection with babesiosis. The key is to quickly decrease the parasitic burden for a good clinical outcome.

Keywords: Babesiosis, Splenectomy, Sepsis

Anahtar Sözcükler: Babesiyozis, Splenektomi, Sepsis 
Conflict of Interest: The author of this paper has no conflicts of interest, including specific financial interests, relationships, and/or affiliations relevant to the subject matter or materials included.

\section{References}

1. Vannier E, Krause PJ. Human babesiosis. N Engl J Med 2012;366:23972407.
2. Haass KA, Sapiano MRP, Savinkina A, Kuehnert MJ, Basavaraju SV. Transfusion-transmitted infections reported to the National Healthcare Safety Network Hemovigilance Module. Transfus Med Rev 2019;33:84-91.

3. Lubin AS, Snydman DR, Miller KB. Persistent babesiosis in a stem cell transplant recipient. Leuk Res 2011;35:77-78.

4. Browne $\mathrm{S}$, Ryan $\mathrm{Y}$, Goodyer M, Gilligan O. Fatal babesiosis in an asplenic patient. Br J Haematol 2010;148:494.

5. Zhao Y, Love KR, Hall SW, Beardell FV. A fatal case of transfusion-transmitted babesiosis in the state of Delaware. Transfusion 2009;49:2583-2587.

๑Copyright 2019 by Turkish Society of Hematology

Turkish Journal of Hematology, Published by Galenos Publishing House

\section{Isolated Mediastinal Myeloid Sarcoma after NPM1-Positive Pediatric Acute Myeloid Leukemia}

\section{NPM1-Pozitif Pediatrik Akut Myeloid Lösemi Sonrası Izole Mediastinal Myeloid Sarkom}

(D) Özlem Tüfekçi, (D) Şebnem Yılmaz, (D) Melek Erdem, (D) Birsen Baysal, (D) Hale Ören

Dokuz Eylül University Faculty of Medicine, Department of Pediatric Hematology, Izmir, Turkey

To the Editor,

Myeloid sarcoma (MS) is a rare extramedullary mass that consists of immature myeloid cells. The most common locations are the soft tissue, bone, periosteum, orbit, and lymph nodes $[1,2]$. Mediastinal involvement is very rare and most commonly reported with concurrent bone marrow involvement [3]. Herein we present a previously treated nucleophosmin (NPM1)-positive acute myeloid leukemia (AML) patient who later presented with isolated mediastinal MS.

A 9-year-old female patient presented with fatigue and weakness. Physical examination revealed no pathological findings. Blood tests demonstrated hemoglobin of $12.2 \mathrm{~g} / \mathrm{dL}$, hyperleukocytosis $(100,500 / \mu \mathrm{L})$, and thrombocytopenia $(43,000 /$ $\mu \mathrm{L})$ with $88 \%$ blasts in the peripheral blood smear. Bone marrow aspirate revealed $90 \%$ blasts with M1 subtype. Treatment was started according to the AML-BFM 2012 protocol. Conventional cytogenetic analysis failed due to lack of spontaneous mitosis and fluorescent in situ (FISH) analysis for $\mathrm{t}(8 ; 21)$, inv(16), $t(15 ; 17)$, and $t(9,22)$ from bone marrow samples revealed negative results. Molecular genetic analysis in the peripheral blood showed NPM1 positivity and FLT3-ITD negativity. Morphologic and molecular remission was obtained at the end of the first induction block. She presented with back pain and fever seven months after cessation of maintenance treatment. Computed tomography (CT) of the thorax showed a solid mass of $84 \times 75 \times 41 \mathrm{~mm}$ in the anterior mediastinum (Figure 1). Bone marrow examination was normal; however, peripheral blood showed NPM1 positivity. Conventional cytogenetic analysis from the bone marrow was within normal limits, while NPM1 could not be studied from bone marrow. Her previous CT scans that were performed for investigation of invasive pulmonary aspergillosis were all normal. Fine-needle aspiration biopsy of the mass was performed; histopathological examination revealed myeloblasts that were positive for myeloperoxidase, CD15, and CD33. Microscopic examination of the imprint of the biopsy also revealed myeloblasts of M1 subtype (Wright stain). Major reduction in tumor mass ( $7 \mathrm{~mm}$ residual tumor) and NPM1 negativity were achieved after one block of FLAG (fludarabine, cytarabine, filgrastim) and two blocks of FLAGmitoxantrone. The patient underwent successful bone marrow transplantation from a matched unrelated donor and has been in remission for one year.

MS of the mediastinum is very rare; most of the cases have been reported as initial presentation with concurrent bone marrow involvement $[3,4,5]$. MS as a relapse has been more frequently reported in post-transplant patients compared to those treated without allogeneic hematopoietic stem cell transplantation [6,7]. Our patient is unique as she presented with isolated mediastinal MS after chemotherapy treatment. Another important point about our patient is that the NPM1 positivity was detected at 